\section{Olaparib shows significant progression-free survival benefit in phase III SOLO2 trial}

The presentation of Study 19 showed that maintenance monotherapy with the poly (ADP ribose) polymerase inhibitor (PARP-I) olaparib impressively prolongs progression-free survival (PFS) in patients with platinum-sensitive recurrent serous ovarian cancer (PSOC) [1] and generated considerable expectation for this first representative of a new class of cancer agents. However, the initial enthusiasm was more among gynecologic oncologists and less with the producing company, which did not take the advantage of being first, relinquishing the lead for further development to niraparib, which confirmed in the phase III NOVA trial that PARP-Is in a pure maintenance setting are able to increase PFS in BRCA-mutated PSOC [2]. Moreover, niraparib demonstrated efficacy regardless of the presence or absence of BRCA mutations or homologous recombination deficiency (HRD) status.

However, there was more to come for olaparib. At the 2017 Society of Gynecologic Oncology 48th Annual Meeting on Women's Cancer the results of the SOLO2 trial were presented [3]. SOLO2 is a phase III randomized study of olaparib maintenance therapy in BRCA-mutated PSOC of very similar design to Study 19. SOLO2 showed a statistically significant improvement in investigator-assessed PFS. The median PFS was 19.1 months in the olaparib arm, compared to 5.5 months in the placebo arm (hazard ratio [HR], 0.30; $<<0.0001$ ). Furthermore, treatment with olaparib increased the time to first subsequent treatment and improved time to second progression (PFS2), demonstrating a continued and consistent clinical benefit beyond progression for olaparib. Based on SOLO2 phase III study, olaparib's new tablet formulation also recently received approval by FDA, as maintenance treatment for women with platinum-sensitive recurrent ovarian cancer, regardless of BRCAmutation status.

SOLO2 and NOVA both show that PARP inhibition has opened new horizons in the treatment of BRCA-mutated PSOC. PFS results are almost superimposable for both PARP-Is with, however, some differences in toxicity. Unfortunately, in contrast to Study 19, SOLO2 did not include BRCA wild-type patients. For those patients gaining greatest benefit, both trials confirm the undoubted role of PARP-Is, whereas, for the others, namely those lacking a germline BRCA mutation, NOVA only enrolled enough patients to allow speculation about a role for PARP-Is in all patients with PSOC.

A significant group of patients are long-term responders, and in both NOVA and SOLO2 approximately $50 \%$ patients remained on PARP-Is after 18 months. Preliminary data suggest that BRCA2 mutations were enriched among those high-responders, whereas BRCA methylation was not associated with response duration [4]. This concept warrants further exploitation. However, as we have learned from the NOVA trial,

\section{Correspondence to:}

Christian Marth, MD, PhD,

AGO-Austria and Department of Obstetrics

and Gynecology, Medical University

Innsbruck, Anichstrasse 35 A-6020

Innsbruck, Austria.

Phone: +43 51250423051

Fax: +4351250423055

E-mail: christian.marth@i-med.ac.at

CANCER BREAKING NEWS 2017;5(2):3-4

DOI: $10.19156 / \mathrm{cbn} .2017 .0041$ 
commercially available HRD status tests might not yet be able to precisely identify patients likely to gain benefit from PARP-Is.

When we assess the efficacy of a new agent, HR is one of our preferred measures of comparative efficacy. Although this is a common approach, is it really relevant to compare HR from different trials? HR in trials testing angiogenesis inhibitors in a similar PSOC population were 0.48 for OCEANS, 0.56 for ICON6 and 0.61 for GOG213. On the other hand, reported HR for PARP-Is were 0.27 and 0.30 for BRCA mutated patients in NOVA and SOLO2 respectively, but 0.45 for patients without germline BRCA mutation in the NOVA trial. Can we use this to confirm the superiority of PARP-Is? This might be a risky comparison. In the PARP-Is trials, patients were pretreated with chemotherapy and randomized only after having a response. On the contrary, in the angiogenesis inhibitor trials patients were randomized before chemotherapy was initiated and irrespective of response to treatment. PARP-Is trials had the advantage of excluding all non-responders and beginning the trial after a test-phase of chemotherapy. It is, therefore, no surprise that the PFS for placebo is around 5 and 10 months in PARP-Is and angiogenesis inhibitor trials, respectively. Consequently, it is impossible to compare trials with completely different designs. However, there is no doubt that PARP-Is have arrived to stay. The ongoing trials testing PARP-Is upfront, such as PAOLA-1 (ClinicalTrials.gov Identifier: NCT02477644) or PRIMA (ClinicalTrials.gov Identifier: NCT02655016), are steps in the right direction to introduce such effective agents into the frontline. It will, however, not remain a SOLO for one PARP-I.

\section{Christian Marth}

AGO-Austria and Department of Obstetrics and Gynecology Medical University Innsbruck, Innsbruck, Austria

\section{Acknowledgments}

The Author thanks Ray Hill, an independent medical writer, who provided native English editing and journal styling on behalf of HPS. This editorial assistance was funded by PharmaMar, Spain.

\section{Conflicts of Interest}

The Author declares there are no conflicts of interest in relation to this article.

\section{References}

1. Ledermann J, Harter P, Gourley $\mathrm{C}$ et al. Olaparib maintenance therapy in platinum-sensitive relapsed ovarian cancer. N Engl J Med 2012;366(15):1382-92.

2. Mirza MR, Monk BJ, ENGOT-OV16/NOVA Investigators et al. Niraparib maintenance therapy in platinum-sensitive, recurrent ovarian cancer. N Engl J Med 2016;375(22):2154-64.

3. Pujade-Lauraine E, Ledermann JA, Penson RT et al. Treatment with olaparib monotherapy in the maintenance setting significantly improves progression-free survival in patients with platinum-sensitive relapsed ovarian cancer: Results from the phase III SOLO2 study [Abstract]. Gynecol Oncol 2017;145(Suppl. 1):219-20.

4. Lheureux S, Lai Z, Dougherty BA et al. Long-term responders on olaparib maintenance in high-grade serous ovarian cancer: Clinical and molecular characterization. Clin Cancer Res 2017 doi: 10.1158/1078-0432.CCR-16-2615. 